\title{
The Realization and Simulation of the Wireless Ad-hoc Network
}

\author{
Xin Xie and Yixin Yan ${ }^{*}$ \\ Harbin University of Science and Technology, Harbin, 150080, P. R. China \\ 'hdxiexin@sina.com, shiyu_wang@126.com
}

\begin{abstract}
The technology of wireless sensor network is a new direction in the field of information science. With the development of science and technology, in modern life, its application is more and more widely. The technology is usually used for information communication in a wide range of network. Sensor network communication can be completed without interference. But the communication process of sensors in some special interferences, such as high-rise block between the sensor or wireless signal interference in the space, communication between wireless sensor network and control terminal may be interrupted. This paper proposes an efficient, stable self-organizing network protocol algorithm in the situation, to realize self organization and information communication. Using STM32 microcontroller as network nodes, it achieves a low power, high speed, high performance wireless ad hoc network, the node and terminal can form a stable network communication, quality of the network communication can be further improved. And compared with the AODV by using NS2, verified the feasibility of routing protocol in this paper.
\end{abstract}

Keywords: Wireless network; self organization; microcontroller; sensor; NS2

\section{Introduction}

The wireless network is composed of a group of mobile nodes with wireless transceiver components, it is a self-organizing and multi hop network. Compared with other communication networks, wireless ad hoc network has no center, self-organizing, multi hop routing, dynamic topology and other characteristics. It has no cable fixed base and each node can be used as a terminal and routing which can switch fast and movie at any time.

It is necessary to keep the communication with other nodes for the nodes in wireless network, so the design of nodes should have a low power consumption and strong communicating ability. STM32 microcontroller is based on ARM Cortex-M3. It is designed for embedded applications wich require high performance, low cost, low power. Using the ARM chip as the node design, nodes can be suitable for various external facilities, and have strong develop ability. It will be more extensive used. This paper mainly describes the use of ARM MCU process from the set of functions of wireless network.

\section{Design Principle}

This design uses STM32F103 MCU and does not need the complex hardware circuit. It will send and receive data by the serial port 2 which is connected with the wireless module. The design realizes the network between the multi nodes and terminal by using a selfdesigned algorithm. It includes a terminal and a plurality of nodes in the system. The terminal is always open. It will receive and deal with the data sending from nodes, then responds to the nodes. It can also sending commands by the serial port 1 connected with the PC. After tuning on the power, the node will connect with the terminal automatically. If the connection is failed, the node will try to connect with other nodes which are in the network. If successes, it 
will transfer data and help the nodes in the blind spots connecting with the network. Figure 21 is the block diagram of the terminal. Figure 2-1 is the block diagram of the nodes.

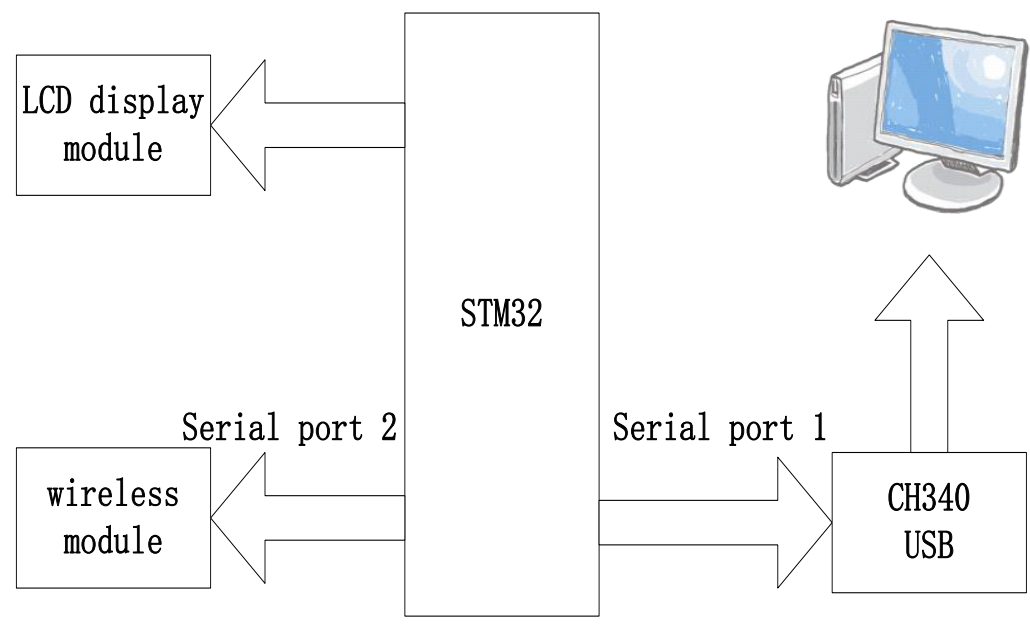

Figure 2-1. The Block Diagram of The Terminal

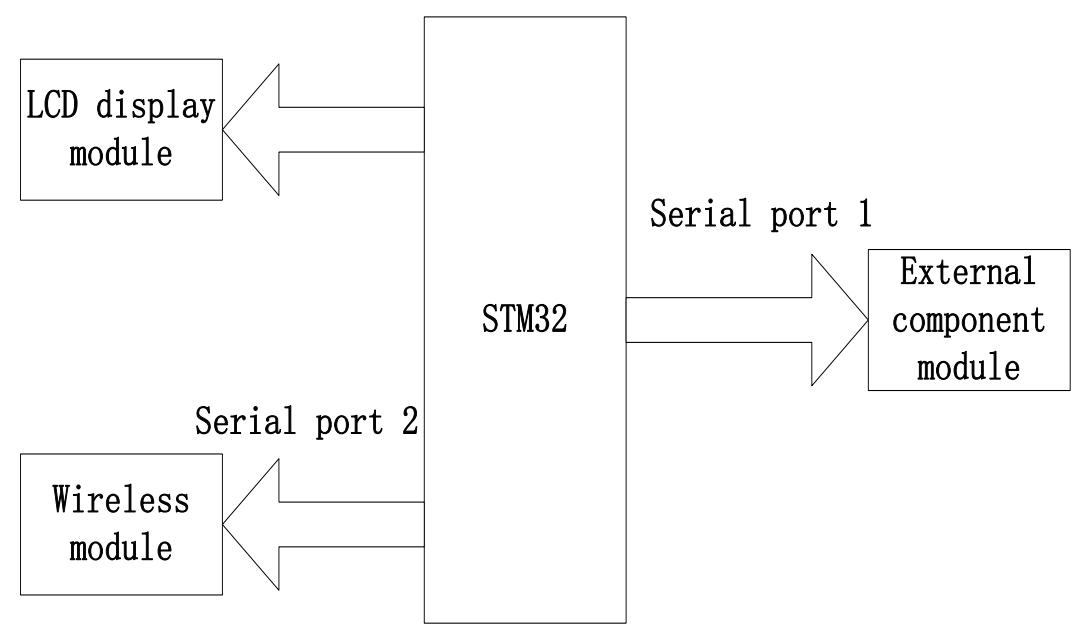

Figure 2-2. The Block Diagram of the Nodes

This algorithm can ensure the communication between nodes and terminal can connect through other routes network when the signal interference happened, and search the blind spot where the nodes cannot reach. Figure 2-3 is the system network structure diagram.

In the diagram, the node $\mathrm{A}$ and node $\mathrm{B}$ can communicate with a terminal network, and node $\mathrm{C}$ is out of the transmission range in the terminal signals but in the the transmission range of the node $\mathrm{A}$ and $\mathrm{B}$. So the node $\mathrm{C}$ can connect with the network through the node $\mathrm{A}$ and $\mathrm{B}$. 


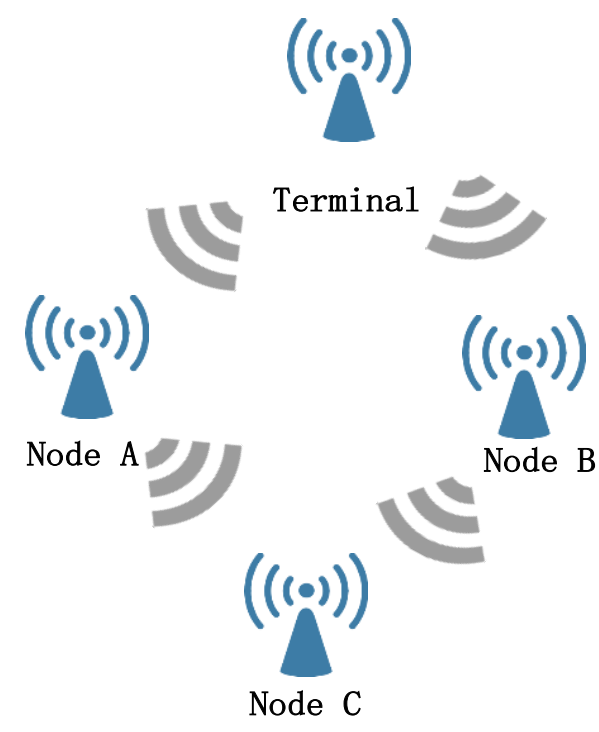

Figure 2-3. Diagram of the System Network Structure Diagram

\section{Self-organization Protocol}

The main challenge of Ad Hoc is that how the distributed routing database saved in the node adapt to the dynamic change of network topology. At present, the typical routing protocol includes AODV, OLSR and DSR, etc.

In this paper, the self-organization routing protocol is improved base on AODV. One is after receiving the routing request, it will treat the node load as the measurement of the responding time. If the node load is smaller, the delay time is shorter. Second is that each node saves the node address in the same link. It reduces the time of transmitting data's and guarantees the success rate of transmission.

\subsection{The Protocol Format}

Self-organization protocol format as follows:

\begin{tabular}{|l|l|l|l|l|l|l|}
\hline START & ID & Aim & Local & Command & Check & END \\
\hline
\end{tabular}

Among them, START is data start mark bit; ID represents the identity, used to distinguish different case data, nodes will make corresponding processing operation according to different identity; Aim indicates that the target address data; Local is the source address, nodes send data content itself; Command represents the data command, the single-chip microcomputer according to the corresponding command execution program; Check is inspection mark, can judge whether the node in the network, to avoid making mistake operation; END indicates the end of data flag.

\subsection{Network Connect Process}

The network includes a terminal connecting with PC and multiple nodes. After tuning on power of terminal and the initialization, enter the mode waiting for the command. Nodes can be arbitrarily placed, after power on and initialization, nodes firstly send a request command of connecting with the network to the terminal, if the terminal confirmation command is received, it have been accessed to and do the operation in the network. If in the set time, it 
can't receive terminal confirm command, that node thinks itself as the blind spot, the node will send the request to the other network node, if the received the node confirm command, have been in the network and saved the address of node. Other node network courses are the same, each node can mount a number of node, the networking can send commands to every node. Self-organization process is shown in Figure 3-1.

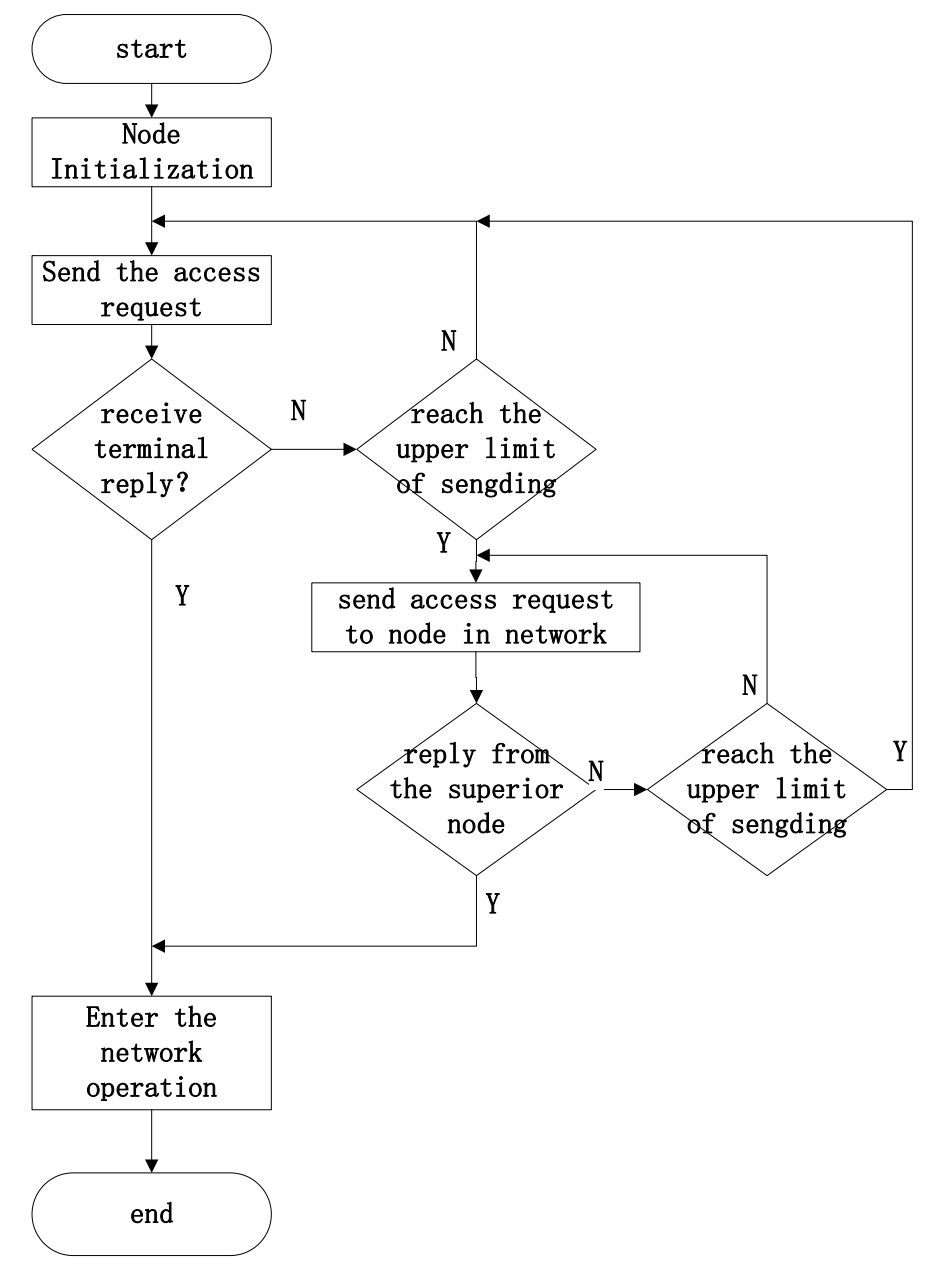

Figure 3-1. Self-Organization Process

\section{The Experimental Simulation and Analysis}

In this paper, it makes the network simulation by using the NS2, and compares the simulation result with AODV. It concludes the performance of the protocol. The experimental simulation results are shown in Figure 4-1, 4-2, 4-3. This paper chooses three common indexes which are used in routing protocol performance: Packet Delivery Ratio, Average Delay, and Routing Load, to evaluate the network performance. 


\subsection{Establishment of Simulation Environment}

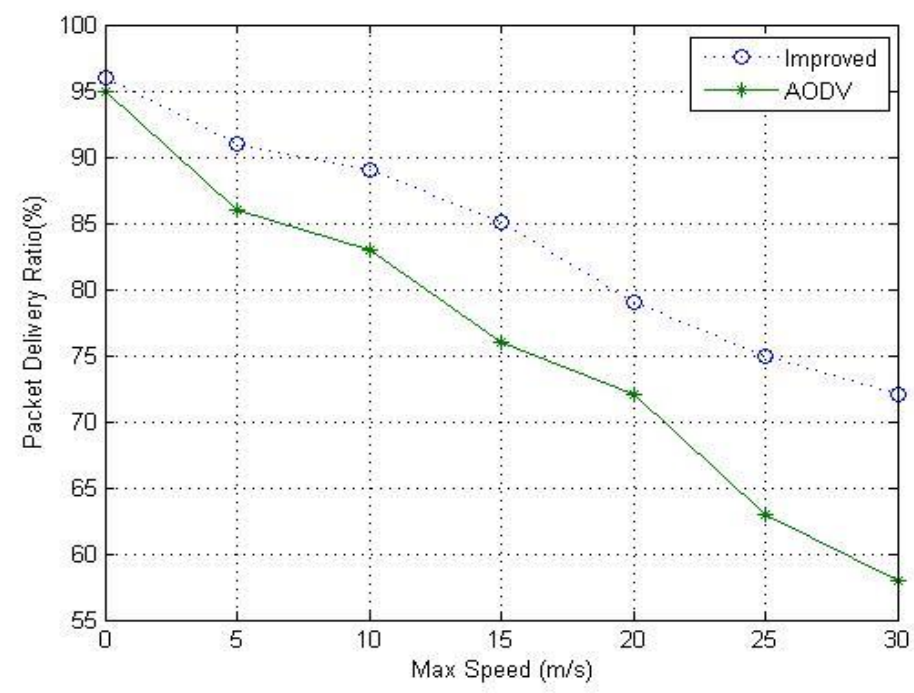

Figure 4-1. Packet Delivery Ratio

According to the result of experiment, when the node's max speed is low, the packet delivery ratio in this paper is higher than AODV. Because when choosing route, it will select the node which has lighter load, so that reducing the probability of discarded packets. With the increase of the max speed, the node in the network may be disconnected, so the node in the disconnected has to save the data, thus it increases the probability that the packet will be lost when the node's cache queue is heavy, causing the packet delivery ratio drop. When the max speed is high, the packet delivery ratio of the routing protocol in this paper is higher than AODV, and drop slowly. Because it takes into consideration the node load when finding the routing, thus it greatly reduces the probability of discarding the data.

\subsection{Average Delay}

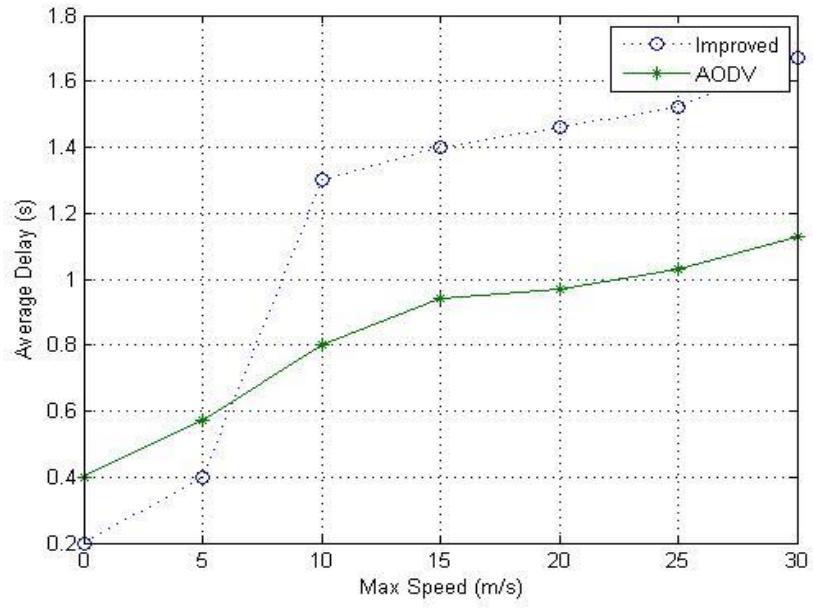

Figure 4-2. Average Delay 
According to the Figure 4-2, we can see when node movement speed is low, in this paper, the routing protocol routing delay than AODV routing protocol. But with the increase of the max speed, the average delay is lower than AODV. Because when sending the routing request, it cannot directly use the node routing cache, only the destination node can return RREP, so increasing the routing request time. When the max speed gets higher, the probability of disconnected increases, the use of AODV cache reduced. The node selection of protocol in this paper is relatively stable, and it reduces the probability of the link broken, so the protocol's average delay is lower than AODV.

\subsection{Establishment of Simulation Environment}

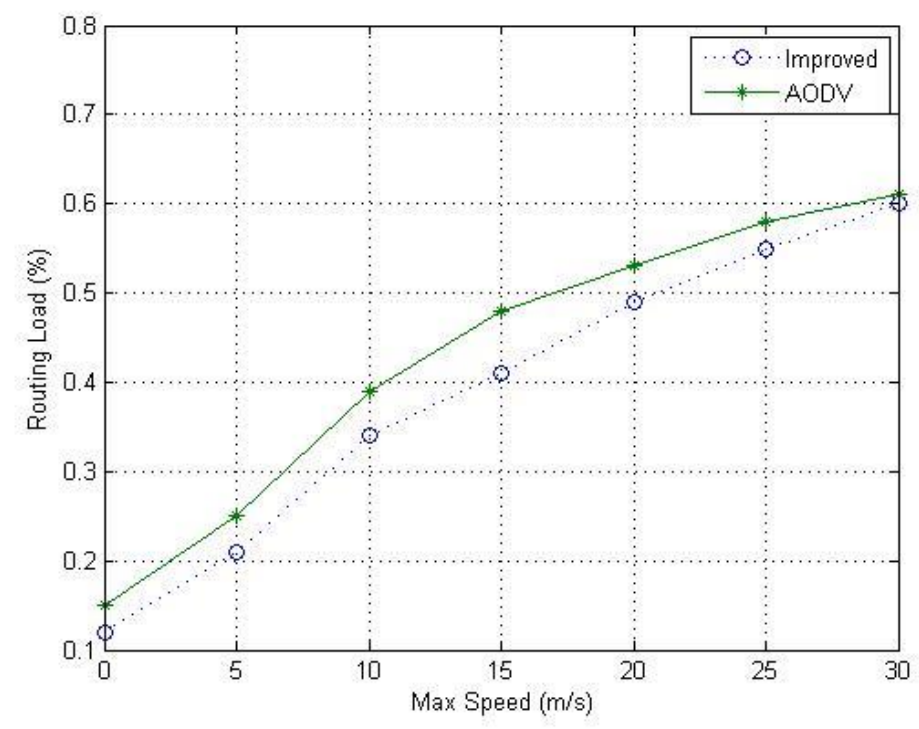

Figure 4-3. Routing Load

According to the Figure 4-3, we can see the routing load of the protocol in this paper is smaller than AODV. For two reasons: one is that the performance of the selected node in this paper is higher, so the routing path repair time caused by the node is small, so reduce the cost of the routing repair. Secondly, when it does the routing repair after the disconnection of the routing link, the cost is less than AODV. Because the AODV scope is bigger when the routing repair, and it will send the RERR to the source nodes which are all through it.

\section{Conclusions}

This paper presents a protocol for wireless ad hoc networks algorithm based on single chip microcomputer. In the self-organization protocol, it will do the logic judgment through the program to receiving data, realize the communication between nodes. The algorithm can optimize transmission route of the network data, and can ensure the correctness of data. The data transmission network is more stable, more efficient. Through practical experiments, this MCU use of wireless ad hoc network protocol can achieve excellent network performance in the case of signal jamming. And compared with the AODV by using NS2, verified the feasibility of routing protocol in this paper. 


\section{Acknowledgement}

This study was financed partially by Higher Education Key Laboratory for Measuring and Control Technology and Instrumentation of Heilongjiang Province, Harbin University of Science and Technology.

\section{References}

[1] K. Sahadevaiah and O. Ramanaiah, “An Empirical Examination of Routing Protocols in Mobile Ad Hoc Networks", International Journal of Communications, Network \& System Sciences, vol. 3, no. 6, (2010).

[2] J. Jubin, J. D Tornow, "The DARPA packet radio network protocols", Proceedings of the IEEE, vol. 75, no. 1, (2010), pp. 21-32.

[3] K. Akkaya and M. Younis, "A survey on routing protocols for wireless sensor network", Ad Hoc Networks, (2005), pp. 25-349.

[4] W. R. Heinzelman, A. Chandrakasan and H. Balakrishnan, "An application-specific protocol architecture for wireless microsensor netwoks", IEEE Transactions on Wireless Communications, vol. 1, no. 4, (2012), pp. 660-670.

[5] R. Mu and W. Zhang, "QAODV: Improved Security Routing Protocol of AODV", Journal of Networks, vol. 8, no. 10, (2013).

[6] R. Yang and Z. Li, "A stablity routing protocols base on Reverse AODV[C]//Computer Science and Network Technology (ICCSNT)", 2011 International Conference, IEEE, (2011), pp. 2419-2423.

[7] F. Tong, W. Tang and L. Peng, et al, "A node-grade based aodv routing protocol for wireless sensor network[C]//Networks Security Wireless Communications and Trusted Computing (NSWCTC)", 2010 Second International Conference, IEEE, (2010), pp. 180-183.

[8] R. Fotohi, S. Jamali and F. Sarkohaki, et al., "An Improvement over AODV Routing Protocol by Limiting Visited Hop Count", International Journal of Information Technology \& Computer Science, vol. 5 , no. $9,(\mathbf{2 0 1 3})$.

[9] X . Cai, J. Li and L . Zhu, "Performance of Routing Protocols in Very Large Scale Wireless Sensor Networks", Journal of Networks, vol. 7, no. 12, (2012).

[10] M. Ayash, M. Mikki and K. Yim, "Improved AODV Routing Protocol to Cope with High Overhead in High Mobility MANETs[C]//Innovative Mobile and Internet Services in Ubiquitous Computing (IMIS)", 2012 Sixth International Conference, IEEE, (2012), pp. 244-251.

[11] K. A. Jalil, Z. Ahmad and J. A. Manan "Securing Routing Table update in AODV routing protocol “, [C], Open Systems (ICOS), IEEE, (2011), pp. 116-121.

[12] K. Jalil, Z. Ahmad and J. L. Ab Manan, "An Enhanced Route Discovery Mechanism for AODV Routing Protocol", [M], Software Engineering and Computer Systems", Springer Berlin Heidelberg, (2011), pp. 408-418. 
International Journal of Future Generation Communication and Networking Vol.7, No.5 (2014) 